\title{
Trigger finger - sonographic diagnostic and therapeutic approach
}

\author{
Florian Berghea \\ "Sf. Maria" Clinical Hospital, "Carol Davila" University of Medicine and Pharmacy, Bucharest, Romania
}

\begin{abstract}
Trigger finger is a very frequent problem identified by rheumatologists. It comes in association with diabetes mellitus and rheumatoid arthritis but also in patients that put their fingers to high mechanical pressure daily. In the incipient stages is not painful and much more difficult to be recognized - both in this phase and in the subsequent, the MSUS offer valuable diagnostic information. Corticoid injection in the affected area represents the best option for trigger fingers with recent onset. The technique should be done under US guidance to maximize the results. Surgery and other conservative options might not possess some benefit to risk ratio in this case.

Keywords: trigger finger, sonographic diagnostic
\end{abstract}

\section{INTRODUCTION}

Trigger finger represents a very frequent hand problem clinically described as a painful popping of the affected finger during flexion and extension. It is found about 3\% of general population (1) with a clear preference for women that are up to six time more frequently affected $(2,3)$. The pathological mechanism involves an increasing conflict between the flexor tendon and the retinacular sheath; the last one becomes inflamed and lately hypertrophied producing an increasingly difficult gliding of the tendon. The retinacular sheath creates so called pulleys (annular and cruciform) that solidarize bones to flexor tendons. First annular pulley (A1) is the most frequent structure involved in trigger finger pathology but is not unusual to discover similar situations in $\mathrm{A} 2$ or A3 (4). Anamnestic is highly probable to discover mechanical overload of the flexors of fingers - is not a matter of the amount of force that overload but about the discrepancy between tendon size and total force applied. The same dysbalanced equilibrium between the force applied and the size (and resistance) of the flexor tendon explains why A1 has to resist to the highest amount of pressure compared with the rest of pulleys.

The disease appears in mature and aged patient being promoted by several comorbidities like diabetes mellitus, rheumatoid arthritis or hypothyroidism.
Several situations are closely related like repetitive trauma (5) and extensive gripping activities $(6,7)$ being most frequent in the dominant hand (8). In diabetic persons, it is better correlated with the total duration of disease than with inappropriate control of blood glucose (9). The ring finger then the thumb is mostly affected (10).

\section{DIAGNOSTIC}

Clinical picture does not involve painful movements at the beginning of disease but just a blockage of normal extension of the first or middle phalanx that is easily solved without external help. Later during disease, the pain appears each time the full extension is produced: first without then with external aid. Looking to avoid the pain, the patient tends to not use the full extension of the involved finger that subsequently produces structural changes of the flexor tendon and finally a fixed flexion position with joint damages (3).

Clinical diagnostic is not difficult especially during the painful popping phase. The imaging techniques have been rarely used in the pre-MSUS (musculoskeletal ultrasound) era: on one hand the X-ray is not useful at all and other techniques like MRI or CT being too expensive.

Ultrasound examination changed the way we understand trigger fingers by showing the conflict be- 


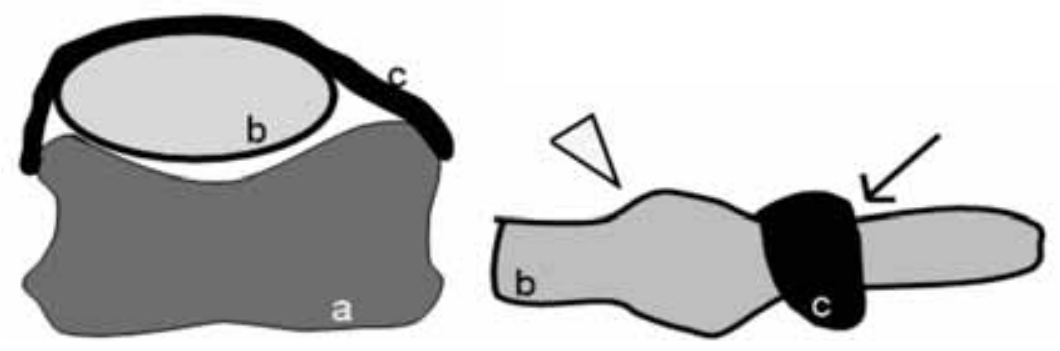

FIGURE 1. Schematic representation of pulley system and trigger finger pathophysiology (left - short axis, right - long axis). a - bone, b-extensor tendon, c-A1 pulley, white arrowhead - increased tendon thickness proximal to the retinaculum, black arrow - injection target point.

tween pulley and tendon both in static and dynamic visualizations. The same technique revealed different pulleys simultaneously involved although the problem was concentrated to only one. Last but not least the MSUS allows more precise conservative therapeutic approaches (opposed to surgery approaches that involve controlled dissection of the affected pulley and releasing of the flexor tendon) (11). The most suitable probes for diagnostic are linear, working on 12 to $18 \mathrm{MHz}$ and having a small footprint. Sonographic appearance of trigger finger was beautifully described like "Madonna face" inspired by "The Madonna of the Yarnwinder" by Leonardo Da Vinci - see Fig. 2, A. Placing the probe in transverse view (short axis), perpendicular on flexor tendon, the pulley is situated about $5 \mathrm{~mm}$ under the skin; it covers the flexor tendon (fibrillar structure and anisotropy sign help the sonographer to identify it). Just deeper the tendon is the bone surface, hyperechogenic, with posterior acoustic shadow. In normal situation the lateral ends of the pulley are just slightly larger than the top portion - see Fig. 2 , B. In trigger finger case the lateral ends are extremely enlarged - see the white arrow, Fig. 2, A. In longitudinal view (long axis) the changes of flexor tendon diameter can be easily observed (12), see Fig.

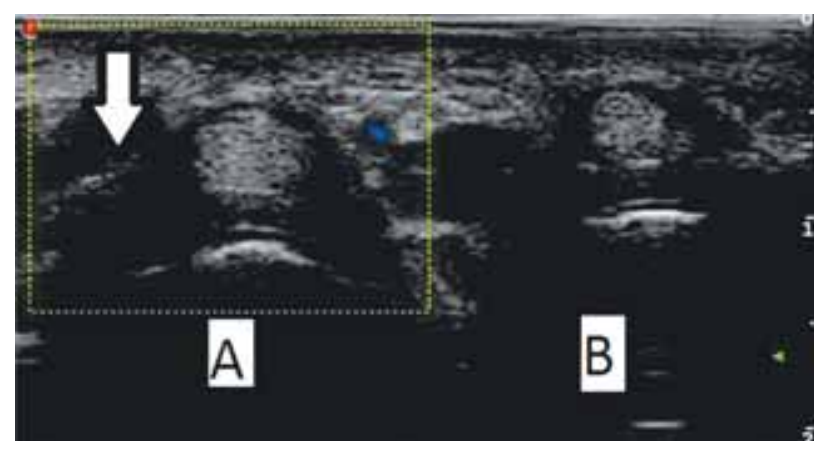

FIGURE 2. Trigger finger - ultrasound view - short axis. A - note the "Madonna image" that announces a hypertrophied pulley (white arrow). B-normal pulley.
1. A diffuse thickening of the synovial sheath is almost always present and so is the irregular internal echotexture (13). Not so rare a cyst attached to the tendon sheath on the volar face could be also observed (13). The measurement of flexor digitorum tendon thickness is usually situated in $0.8 \pm 0.4 \mathrm{~mm}$ range and $\mathrm{A} 1$ thickness falls in $0.8 \pm 0.2 \mathrm{~mm}$ range during the disease (14).

\section{MANAGEMENT}

Therapeutic approaches in trigger finger disease might be conservative but can end to surgical release of the tendon and dissection of the affected pulley (3, 15). The conservative approach involves changes of typical activities performed with the aid of the involved finger, regular doses of NSAIDs, joint immobilization and corticosteroids injections. If patient refuse surgery or corticosteroid injection, then splinting could be an alternative option with positive results in about half of cases (16). Surgical option could produce better results solving the problem in about $90 \%$ of cases (3). However, there are some surgical complications that might appear (3): nerve and artery lesions, reflex sympathetic dystrophy, infection, stiffness, incision pain, flexion deformity, flexor tendon bowstringing, and recurrence (3\%).

Corticosteroid injection is frequently used since 1953 when was firstly announced (17). The success rate is very high (up to 93\%) that places this technique ahead the surgery option (18). It is particularly successful in recent onset diseases (less than 6 moths), non-diabetic persons, only one pulley involved (in just one finger) (19). The aim is to place some amount of corticosteroids in the tendon sheath but some authors described good results when the steroid is placed in the near vicinity (20). In 3 weeks the diameter of flexor tendon decrease to about half of the initial dimensions (flexor tendon: $0.4 \pm 0.3$ 


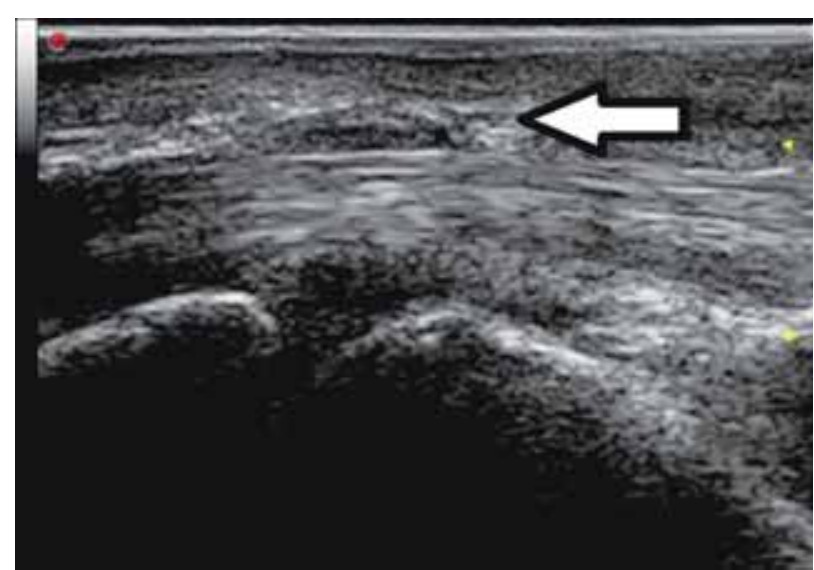

FIGURE 3. Trigger finger - ultrasound view - long axis. Note the enlarged pulley (white arrow)

$\mathrm{mm}$ and A1 pulley: $0.4 \pm 0.3 \mathrm{~mm}$ ) (14). Pain is also very responsive to this treatment with a massive reduction of VAS in the first week after the injection (14): pre-injection, $7.3 \pm 0.9$; week $1,1.7 \pm 0.4$; week $3,1.2 \pm 0.3$. There is not a general consensus regarding what is the choice drug for this intervention - in our practice we use long lasting products (like Diprophos $\left.{ }^{\circledR}\right)$. The blind technique is less reliable than the ultrasound guided procedure (21) that explain why the last one produces better results.

Our technique is an adaption from the one described by Bodor and Flossman (22) and was per- formed in more than 50 cases. Working in a very narrow area is mandatory to use the full sterile approach: very clean probe, sterile cover for the probe, sterile gel. There are two approaches that can be used - lateral and longitudinal. We prefer the longitudinal one to avoid local nerves and arteries. A very good cleaning of the whole hand of the patient is used; this cleaning is finished with one skin disinfectant generously applied. A sterile cooling aerosol might be applied on the targeted skin area to reduce the pain. Finally, the involved finger is disinfected with iodine solution. A thin needle should be used (26G), if possible a longer one $(15 \mathrm{~mm}$ should be all right but 10 $\mathrm{mm}$ could be used, too). Some practitioners use the needle as it is, other prefer to bend it - in our experience we found more easily to perform the injection with a bended needle - this is not a difficult task as is performed with the sterile needle cup as depicted in Fig. 4. A large amount of sterile gel is needed (see Fig. 5) as long as needle will pass the gel before penetrating the skin. The critical moment is represented by the skin puncture: the volar part of the finger possesses an abundant sensitive innervation and the patient feels a certain amount of pain. The needle is usually visualized when it is at the half of distance between the surface and the tendon ( 2 to $3 \mathrm{~mm}$ ) - see

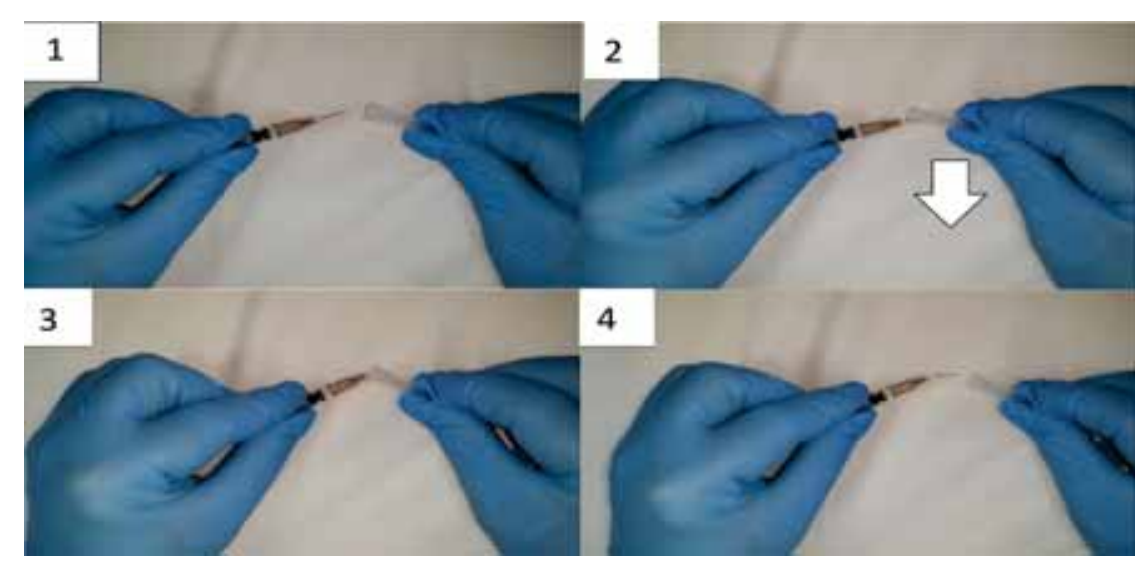

FIGURE 4. Bending the needle for corticoid injection (from step 1 to 4). White arrow indicates the force applied to the sterile needle cap

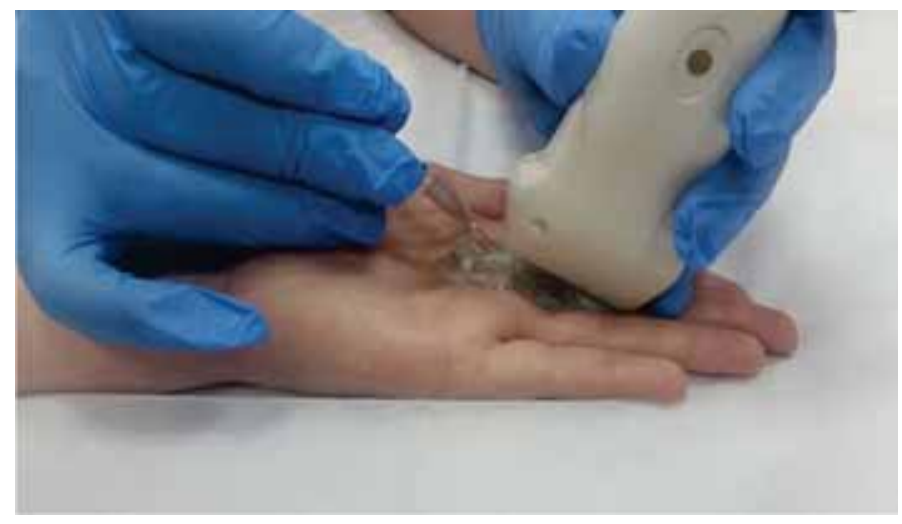

FIGURE 5. Ultrasound guided pulley injection - long axis. Note the large amount of gel used and how the needle pass the sterile gel before penetrating the skin. 


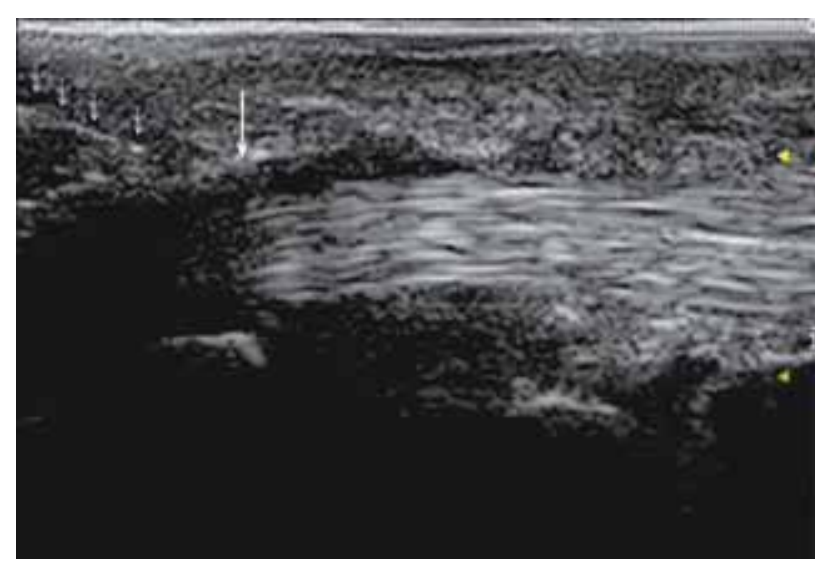

FIGURE 6. Ultrasound view of pulley injection - long axis. Small arrows - needle, large arrow - target area.

Fig. 6. In few more millimeters the tip of the needle reaches tendon sheath and the injection can start. If the needle entered to deep into the tendon the injection is difficult and no corticosteroid could be observed covering the tendon; in this case the needle is gently retracted keeping a small pressure in syringe until the syringe piston start to move easy. There's no need to fear of tendon lesion produced by an unique needle penetration. The total amount of metilprednisolone (retard) injected should be around $1 \mathrm{ml}(40 \mathrm{mg})$ or about $1 \mathrm{ml}(7 \mathrm{mg})$ in case Diprophos ${ }^{\circledR}$ (bethametasone) is used. There are some reports about the usage of hyaluronic acid in combination with corticosteroids with similar good results (15 positive results out of 16 cases) (23). The necessary of a $2^{\text {nd }}$ injection appears in about $17 \%$ of the injected fingers and in $6 \%$ the surgical solution is finally needed (24).

\section{CONCLUSIONS}

Trigger finger represents a frequent problem seen in the rheumatology ward. The musculoskeletal ultrasonography is mature enough to diagnose it and represents an unmatched help for a precise injection of the affected structures.

Conflict of interest: none declared Financial support: none declared

14. Mifune Y., Inui A., Sakata R. et al. Skeletal Radiol (2016) 45: 1661. doi:10.1007/s00256-016-2485-5

15. Ryzewicz M., Wolf J.M. Trigger digits: principles, management, and complications. J Hand Surg (Am) 2006; 31:135-46.

16. Rodgers J.A., McCarthy J.A., Tiedeman J.J. Functional distal interphalangeal joint splinting for trigger finger in laborers: a review and cadaver investigation. Orthopedics 1998; 21:305-9, discussion 309-10.

17. Howard L.D. Jr, Pratt D.H., Bunnell S. The use of compound F (hydrocortone) in operative and non-operative conditions of the hand. J Bone Joint Surg Am 1953; 35:994-1002.

18. Freiberg A., Mulholland R.S., Levine R. Nonoperative treatment of trigger fingers and thumbs. J Hand Surg (Am) 1989;14:553-8.

19. Green D., Hotchkiss R., Pederson W., et al. Tenosynovitis. In: Green's operative hand surgery. 5th ed. London: Churchill Livingstone; 2005.

20. Kazuki K., Egi T., Okada M. et al. Clinical outcome of extrasynovial steroid injection for trigger finger. Hand Surg 2006; 11:1-4.

21. Mardani Kivi M., Lahiji F.A., Jandaghi A.B., Saheb Ekhtiari K., Hashemi M.K. Efficacy of sonographically guided intra-flexoral sheath corticosteroid injection in the treatment of trigger thumb. Acta Orthop Traumatol Turc. 2012;46:346-52.

22. Bodor M., Flossman T. (2009) Ultrasound-guided first annular pulley injection for trigger finger. J Ultrasound Med 28:737-743

23. Callegari L., Spano E., Bini A., Valli F., Genovese E., Fugazzola C. (2011) Ultrasound-guided injection of a corticosteroid and hyaluronic acid: a potential new approach to the treatment of trigger finger. Drugs R D 11:137-145

24. Cecen G.S., Gulabi D., Saglam F. et al. Arch Orthop Trauma Surg (2015) 135: 125. doi:10.1007/s00402-014-2110-9 